\section{Oral cancer - improving early detection and promoting prevention. Are you up to date?}

\author{
A. J. Mighell'1 and J. E. Gallagher ${ }^{2}$
}

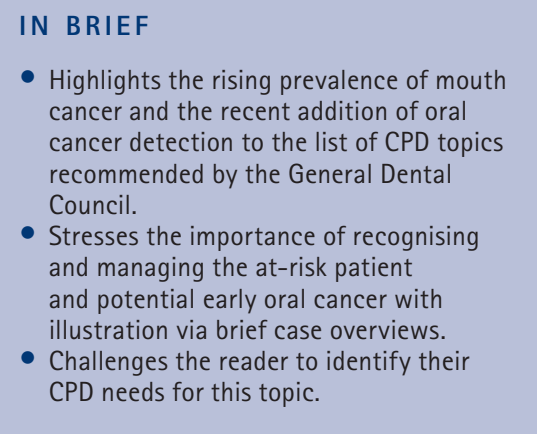

Oral cancer is increasingly common. The need for early detection and promoting prevention is greater than ever and relevant to all who are responsible for the care of patients. Recent addition of oral cancer detection to the list of continuing professional development (CPD) topics recommended by the General Dental Council (GDC) reflects this importance.

\section{INTRODUCTION}

How confident are you about assessing patients' risk of developing oral cancer and picking up cancerous lesions, especially early malignancy? Do you know what to do if you see something unusual? Have you ever had any CPD on improving oral cancer early detection? What actions do you take to identify and manage patient risks of developing oral cancer and promote prevention? Given rising trends in oral cancer, these issues are a priority for all members of the dental team responsible for chair-side patient care. This is reflected by the recent decision by the GDC to add oral cancer detection as a recommended CPD topic before completion of its CPD review. ${ }^{1}$ This decisive action followed lobbying from within and beyond the profession, which included strong representation by Cancer Research UK and underlines the seriousness of this issue.

\section{ORAL CANCER - \\ THE INCREASING PROBLEM}

Oral cancer (OC), including oropharyngeal cancer and cancer of the lips (ICD 10 C00-C06, C09-C10 and C12-C14)

\footnotetext{
${ }^{1 *}$ President of the British Society for Oral Medicine and Senior Lecturer and Honourary Consultant in Oral Medicine, Leeds Dental Institute, Clarendon Way, Leeds, LS2 9LU: ${ }^{2}$ President of The British Association for the Study of Community Dentistry and Reader in Oral Health Services Research and Consultant in Dental Public Health, King's College London Dental Institute, Denmark Hill Campus, Bessemer Road, London, SE5 9RS ${ }^{*}$ Correspondence to: Dr Alan Mighell

Email: a.j.mighell@leeds.ac.uk; Tel: +44 113 3435,688
}

\section{Refereed Paper}

Accepted 30 July 2012

DOI: 10.1038/sj.bdj.2012.838

${ }^{\circ}$ British Dental Journal 2012; 213: 297-299 is one of the few oral diseases encountered by the dental team that can result in significant morbidity and premature mortality.

The age standardised incidence of OC in Great Britain has steadily increased over the last 20 years with a $66 \%$ increase between 1988-90 and 2007-09.* Over the next 20 years $0 \mathrm{C}$ rates are projected to increase by around 1\% per year unless decisive public health action is taken., ${ }^{2,3}$ Of all UK cancers, only melanoma, a skin cancer that may also be recognised by the dental team, is increasing more rapidly. ${ }^{3}$

It has recently been predicted that by 2030 there will be 9,200 cases of OC in the UK per year compared to 6,240 in 2009 and 3,030 in 1984. ${ }^{2,3}$ OC affects both sexes with a male:female ratio of 19:10 in 2009. ${ }^{2}$ The dental team can expect to encounter OC more frequently in the future than in the past or currently.

The brief case overviews illustrate some of the key issues (Table 1 and Figs 1-6). Regrettably, delayed OC diagnosis has resulted in GDC Fitness to Practice hearings. Although as a clinician you may only pick up a few cases of OC in your working life, you want to be ready to do so with confidence and in a timely and professional manner.

\footnotetext{
*This incidence/mortality data was compiled by the statistical information team at Cancer Research UK using data from the regional cancer registries in England, Wales and Scotland. Online information available at http://info.cancerresearchuk.org/cancerstats/.
}

\section{IDENTIFYING THE PATIENT AT RISK AND ACTING ON THIS}

OC is multi-factorial in origin. Recognised risk factors are tobacco, alcohol and areca nut use, infection with human papillomavirus (HPV) and for the external lips, sunlight exposure. ${ }^{4-7}$ OC rates are currently highest in areas of social deprivation, partially reflecting higher levels of tobacco use. However, it is important to remember that $\mathrm{OC}$ occurs in adults from all backgrounds across our society and not all adults present with the common risk factors. The dental team can expect to care for adult patients at risk of $\mathrm{OC}$, irrespective of where they practice.

Recognition of the social and behavioural risk factors for $O C$ brings the responsibility of acting on these and promoting the health benefits of modifying behaviour with documentation in the clinical records. Multi-disciplinary working is often indicated, for example, with respect to tobacco cessation or changing alcohol use. The dental team are wellplaced to promote accurate information about $\mathrm{OC}$ risk factors, not only with respect to their impact on oral health, but also general health.

Many GDC registrants will be aware of key issues related to risk factors for OC including those described in Delivering better health ${ }^{8}$ and similar resources. ${ }^{9-11}$ However, familiarity with other risk factors including HPV may be less complete. Engaging patients effectively in understanding risk factors for OC empowers patients to take responsibility for their 
own health, including seeking early professional advice if new lesions develop.

\section{THE NEED FOR EARLY REFERRAL OF SUSPICIOUS LESIONS}

A diagnosis of $\mathrm{OC}$ and the associated impact of management have enormous implications on day-to-day activities of living such as eating, drinking, speaking, socialising and working. Furthermore, OC five-year survival rates have shown very limited improvement over the last 20 years. ${ }^{12}$ This contrasts with other cancers where significant improvements in survival have been achieved over the same time period. ${ }^{12}$ Just over half the patients diagnosed with tongue or oropharyngeal cancer can expect to be alive after five years. ${ }^{12}$ However, the majority of OCs are potentially curable if diagnosed early.

Systematic examination of the oral soft tissues is a long established practice within oral healthcare. The importance of this is emphasised by the rise in HPV-associated oropharyngeal cancer that typically occurs at sites that are not easily observed by either the clinician or patient. In England, oral health assessment of patients in the current 'pilots' for a new dental contract, includes an assessment of risk and soft tissue examination - the algorithm will advise on the patient's risk denoted by a 'RAG rating' (red, amber and green) and the appropriate action to be taken. The recommended actions are in line with the evidence base for prevention. ${ }^{8,9}$

Oral cancer is managed within head and neck cancer multi-disciplinary teams in selected centres. Mechanisms for rapid referral of suspected head and neck cancer to these teams through the "two-week wait' pathway are well established. Within these guidelines, description is given of the clinical situations that should trigger such a referral. ${ }^{13}$ However, there is evidence that only a quarter of all OCs are diagnosed via this route. ${ }^{14}$ It is the responsibility of the referring clinician to be aware of local care pathways and evaluate whether the patient's situation warrants rapid referral. These decisions need to be fully informed and will be influenced by not only lesion assessment, but also of the patient's circumstances and concerns. In cases of uncertainty, it is best to err on the side of caution and refer via the fasttrack system, while being mindful of the

Table 1 Brief case overviews of OC presentations for six patients. Each illustrates different points, how presentations vary and can be unexpected

\section{Case Presentation and comment}

1 Presentation: 50-year-old with a longstanding, enlarging, ulcerated and indurated lesion involving the lateral tongue that on biopsy was confirmed as squamous cell carcinoma (SCC) (Fig. 1). Comment: A lesion that is $\mathrm{OC}$ until proven otherwise on biopsy.

2 Presentation: 81-year-old with limited spoken English complained of a persistent sore throat and difficulty swallowing. A defensive tongue made examination of the root of the tongue and oropharynx difficult. a) Localised erythema (Fig. 2a) hinted at pathology that b) on depression of the tongue revealed an advanced SCC (Fig. 2b).

Comment: Language barriers can contribute to delays. OCs often arise at sites that are difficult to examine and this is expected to increase with the rise in HPV-associated OC.

3 Presentation: 46-year-old with diffuse red and white changes in the floor of the mouth with a long history of heavy tobacco and alcohol abuse with dental neglect. Biopsy confirmed SCC (Fig. 3).

Comment: An example of widespread oral mucosal change that is $\mathrm{OC}$ until proven otherwise in a patient with clear risk factors. Even though this was explained ahead of the biopsy, the diagnosis was still a surprise to the patient who had been on the internet and concluded that the red and white lesions did not look like OC (typified by a chronic ulcer with rolled margins).

4 Presentation: a) 30-year-old with a white patch involving the right tongue that on biopsy was hyperkeratosis with no dysplasia (Fig. 4a). The patient was advised that smoking 20 cigarettes a day and drinking 80 units of alcohol a week increased the risk of OC. b) On re-presentation 18 months later there was a small, painful ulcer at the same site (Fig. 4b). Biopsy confirmed SCC.

Comment: The patient chose not to significantly modify their tobacco and alcohol habits even though the risks of these were understood and support to make changes was offered. A biopsy report with no worrying features can provide false reassurance, but should not distract from the need to modify risk factors, repeat oral soft tissue examinations over time and avoid delays in future referral.

5 Presentation: a) 26-year-old with painful ulceration of the right tongue that relapsed and remitted and was attributed to trauma from an instanding lower molar tooth (Fig. 5a). The patient smoked ten cigarettes a day for around ten years. b) Over the next six months the ulceration became persistent (Fig. 5b). Biopsy confirmed a SCC.

Comment: Follow-up over time is important and allows early recognition of changing lesions. Biopsy is currently the only way to establish a tissue diagnosis. OC is increasingly prevalent in young adults.

6 Presentation: 66-year-old with a small white patch of the right tongue adjacent to a lonestanding lower molar tooth restored with a gold crown. The patient had never used tobacco and consumed very little alcohol. A working diagnosis of lichenoid reaction was made, but biopsy confirmed a SCC (Fig. 6).

Comment: $\mathrm{OC}$ can develop in the absence of tobacco use and excessive alcohol consumption.
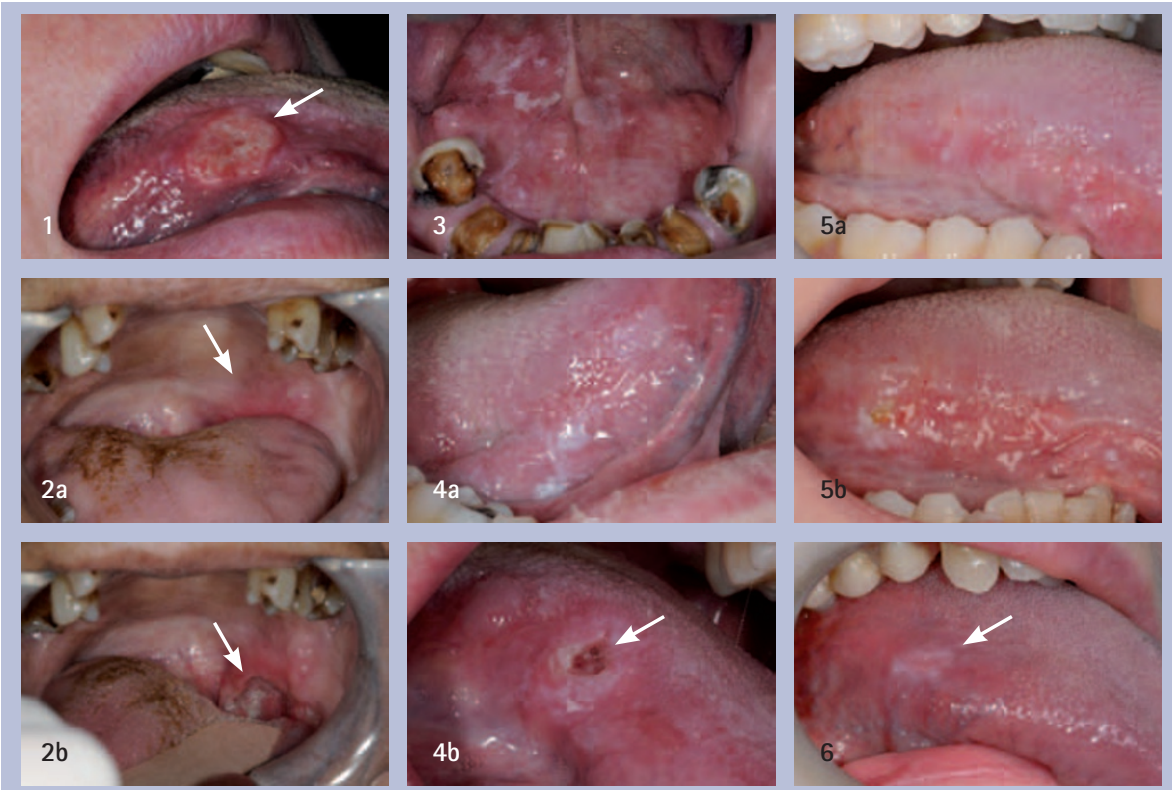

Figs 1-6 Clinical presentations of OC linked to the six cases described in Table 1.

need to use this route responsibly and the impact of any referral on a patient and the NHS. Irrespective of the referral route chosen, it is important to do this in partnership with the patient and provide a clear and realistic explanation as to the purpose of the referral and address their concerns appropriately. 


\section{REVIEW YOUR PRACTICE AND IDENTIFY YOUR PROFESSIONAL DEVELOPMENT NEEDS}

Key issues around OC early detection and the importance of promoting prevention have been highlighted. A wide range of CPD opportunities already exist via employers, commissioners, professional associations, postgraduate deaneries and commercial CPD providers to help you keep up-to-date. All GDC registrants responsible for chair-side patient care have an opportunity, and responsibility, to make a difference. This includes identifying and addressing risk factors as well as identifying and managing referral of possible or frank malignancy in a timely and professional manner.

The authors thank Hazel Nunn, Head of Health Evidence and Information, Cancer Research UK, for her comments and input.
1. General Dental Council. Announcement: oral cancer-improving early detection becomes recommended CPD. London: GDC, 2012. Online announcement available at http://www.gdc-uk. org/Newsandpublications/Pressreleases/Pages/ Oral-Cancer---Improving-Early-Detection-.aspx (accessed August 2012).

2. Cancer Research UK. Oral cancer-UK incidence statistics 2009/10. Online statistics available at http://info.cancerresearchuk.org/cancerstats/types/ oral/ (accessed August 2012).

3. Mistry M, Parkin D M, Ahmad A S, Sasieni P. Cancer incidence in the United Kingdom: projections to the year 2030. Br J Cancer 2011; 105: 1795-1803.

4. Cancer Research UK. Smoking statistics. Online statistics available at http://info.cancerresearchuk.org/ cancerstats/types/lung/smoking/ (accessed August 2012).

5. NHS Information Centre. Health survey for England 2009: health and lifestyles. London: The Health and Social Care Information Centre, 2010. Online article available at http://www.ic.nhs.uk/pubs/hse09report (accessed August 2012).

6. Syriänen S. The role of human papillomavirus infection in head and neck cancers. Ann Oncol 2010; 7: 243-245

7. Warnakulasuriya S. Causes of oral cancer - an appraisal of controversies. Br Dent J 2009; 207: 471-475.

8. Department of Health, the British Association for the Study of Community Dentistry. Delivering better oral health: an evidence-based toolkit for prevention - second edition. London: $\mathrm{DH}$ and BASCD, 2009. Online article available at http://www. dh.gov.uk/en/Publicationsandstatistics/Publications/ PublicationsPolicyAndGuidance/DH_102331 (accessed August 2012).

9. Scottish Intercollegiate Guidelines Network (SIGN) Diagnosis and management of head and neck cancer. A national clinical guideline. Edinburgh: SIGN; 2006. Online article available at http://www.sign. ac.uk/pdf/sign90.pdf (accessed August 2012).

10. NHS Direct Wales. Cancer of the mouth. Online encyclopaedia available at http://www.nhsdirect. wales.nhs.uk/Encyclopaedia/c/article/cancerofthemouth/ (accessed August 2012)

11. Cancer Research UK. Oral cancer prevention. Online information available at http://info.cancerresearchuk.org/cancerstats/types/oral/prevention/ oral-cancer-prevention (accessed August 2012).

12. Cancer Research UK. Oral cancer-survival statistics for England and Wales. Online information available at http://info.cancerresearchuk.org/cancerstats/ types/oral/survival/ (accessed August 2012).

13. National Institute for Clinical Excellence. Referral guidelines for suspected cancer. http://publications. nice.org.uk/referral-guidelines-for-suspectedcancer-cg27 (accessed August 2012).

14. National Cancer Intelligence Network. Routes to diagnosis- NCIN data briefing. UK: NCIN, 2010. Online information available at http://ncin.org.uk/ publications/data_briefings/routes_to_diagnosis. aspx (accessed August 2012). 